\title{
DETECTION OF DIABETIC RETINOPATHY BY SCANNING LASER OPHTHALMOSCOPY
}

\author{
W. N. WYKES, A. A. E. PYOTT and V. G. M. FERGUSON \\ Glasgow'
}

\begin{abstract}
SUMMARY
A scanning laser ophthalmoscope (SLO) was used to examine the fundi of $\mathbf{5 4}$ diabetic patients through undilated pupils and the results compared by an experienced ophthalmologist with clinical examination through dilated pupils to assess the effectiveness of the SLO in detecting diabetic retinopathy. Whilst the SLO was not as good at detecting cotton wool spots and subtle intraretinal microvascular abnormalities, it did not miss any active new vessel formation and all eyes needing treatment would have been referred.
\end{abstract}

Diabetic retinopathy accounts for $7-8 \%$ of blind registrations in the United Kingdom and is the commonest cause of blindness in the working population,' despite improved detection and treatment of patients with diabetic retinopathy. The question of how and by whom diabetic patients should be screened has long been debated. ${ }^{2.3}$ Direct ophthalmoscopy, indirect ophthalmoscopy, slit lamp examination with a fundus contact lens or a 90 dioptre lens, non-mydriatic photography, fluorescein angiography and colour fundus photography through dilated pupils have all been tried with the involvement of ophthalmologists, diabetic physicians, general practitioners, optometrists and technicians. ${ }^{2-7}$ In this prospective study the relatively new technique of scanning laser ophthalmoscopy was compared with a conventional clinical examination by an ophthalmologist.

The scanning laser ophthalmoscope (SLO) uses a focused laser beam (a helium-neon laser in the Rodenstock SLO used in this study) which is directed in a raster format across the retina. ${ }^{8}$ A digitised video image is generated by collecting reflected light for one point on the retina at a time, the signals being displayed as points of varying intensity on a video monitor. The SLO provides highresolution images of the fundus without requiring the high levels of incident illumination used in conventional funduscopy.

Correspondence to: Dr W. N. Wykes, The Eye Department, Southern General Hospital, 1345 Govan Road, Glasgow G51 4TF, UK.

\section{METHODS}

Patients were recruited into the study from the diabetic eye clinic over a 6 month period. They were excluded if they were not mobile, or were unwilling to participate in the study. Both new and review patients were included in the study. Some patients had already had laser therapy.

All patients underwent scanning laser ophthalmoscopy whilst their pupils were undilated. This was performed by an experienced ophthalmic photographer who recorded views of the optic discs, maculae and peripheral retina to include a $60^{\circ}$ field, using the helium-neon laser of the SLO, recording onto U-Matic videotape. Following mydriasis with $\mathrm{g}$. tropicamide $1 \%$ and $\mathrm{g}$. phenylephrine $10 \%$, the patients underwent clinical examination with indirect ophthalmoscopy, slit lamp examination with a 90 dioptre lens and, where appropriate, direct ophthalmoscopy.

The SLO images were reviewed independently by one ophthalmologist (A.A.E.P.) and were then scored on a chart and the features of background, preproliferative, proliferative retinopathy and maculopathy noted. The clinical findings were scored on identical charts by another ophthalmologist (W.N.W.). Background retinopathy included exudates, dot and flame haemorrhages and microaneurysms. Preproliferative changes were divided into cotton wool spots, intraretinal microvascular haemorrhages (IRMA) and venous beading. Proliferative retinopathy was recorded for eyes with new vessels at the optic disc (NVD) or elsewhere (NVE). If vitreous haemorrhage was noted this was recorded. There were no patients in the study with a retinal detachment. Maculopathy was divided into three groups: focal exudative, where circinate retinopathy was present; diffuse exudative, with a significant number of exudates but not in a specific circinate pattern; and diffuse oedematous, with slight oedema and loss of foveal detail but without significant exudates. Cystoid macular oedema (CMO) was specifically noted. The two sets of charts were then compared. Where discrepancies were noted the clinical findings were reviewed to see whether there were obvious explanations for the differ- 
ence. All patients have been reviewed for at least 1 year after inclusion in the study.

\section{RESULTS}

One hundred and eight eyes of 54 patients were examined by the two techniques. In 5 eyes the SLO images were too poor to interpret, and in a further 7 the images were poor but usable (Table I). Cataracts prevented a view of the fundus in 2 eyes, and in another 2 eyes (of 1 patient) made the image poor, but in the latter case the SLO image was more useful than the clinical view. In 4 eyes of 2 patients the pupils were too small to allow an adequate view with the SLO, but they had clear media and it is likely that an adequate assessment could have been made after dilatation with tropicamide alone.

The results for the remaining 103 eyes are shown in Table II.

Background retinopathy. In 76 of 82 eyes (92.7\%) the two techniques were in agreement. Sixty-five of these had no other retinopathy. The SLO failed to detect retinopathy in 5 eyes, but in 3 of these it was minimal.

Preproliferative retinopathy. There was agreement for 5 eyes. This includes 1 eye in which the SLO suggested new vessels but the same area was recorded as IRMA clinically, and has been recorded as a preproliferative change (IRMA) seen by both techniques. On further review this area has remained static. Clinical examination missed preproliferative signs in 3 eyes, 2 of which had venous beading, a sign which is clearly detected on the high-contrast black and white SLO images. The SLO failed to detect signs in 8 eyes, 5 of which had cotton wool spots, which do not show up well, and 3 of which had IRMA.

Proliferative retinopathy. There was agreement between the two techniques for 7 cases of proliferative change. The SLO missed 4 cases of new vessels at the optic disc, but on checking the clinical data 3 of these were inactive vessels following laser treatment, and the fourth had a vitreous haemorrhage. The SLO missed 1 area of new vessels elsewhere, but these too were inactive.

In 2 cases the SLO proved superior at detecting proliferative retinopathy. In 1 eye a cataract precluded a clinical view of new vessels at the optic disc, but these were detected with the SLO. After urgent cataract surgery the new vessels were confirmed clinically. In another eye, NVE were seen with the SLO alone. At the next review, the vessels were noted clinically.

Maculopathy. There was agreement for 27 of 34 (79.4\%) eyes. The SLO missed 5 cases of exudative maculopathy,

Table I. Reasons for poor scanning laser ophthalmoscope images

\begin{tabular}{lll}
\hline & $\begin{array}{c}\text { Unusable } \\
\text { (no. of eyes) }\end{array}$ & $\begin{array}{c}\text { Poor but usable } \\
\text { (no. of eyes) }\end{array}$ \\
\hline $\begin{array}{l}\text { Cataract } \\
\text { Vitreous haemorrhage }\end{array}$ & 2 (1 patient) & 2 (1 patient) \\
Patient too mobile & 2 (1 patient) & 0 \\
Pupil too small & 0 & 4 (2 patients) \\
Total & 5 & 7 \\
\hline
\end{tabular}

Table II. Comparison of scanning laser ophthalmoscope funduscopy with conventional clinical examination in detecting diabetic retinopathy

\begin{tabular}{|c|c|c|c|}
\hline & SLO = Clinical & Clinical missed & SLO missed \\
\hline Nil & 22 & & \\
\hline Background & $76^{a}$ & 1 & $5^{\mathrm{b}}$ \\
\hline \multicolumn{4}{|l|}{ Preproliferative } \\
\hline CWS & 1 & 1 & 5 \\
\hline IRMA & 1 & 0 & 3 \\
\hline Beading & 3 & 2 & 0 \\
\hline \multicolumn{4}{|l|}{ Proliferative } \\
\hline NVD (active) & 2 & 1 & $1^{\circ}$ \\
\hline NVE (active) & 5 & 1 & 0 \\
\hline NVD (inactive) & 0 & 0 & 3 \\
\hline NVE (inactive) & 0 & 0 & 1 \\
\hline \multicolumn{4}{|l|}{ Maculopathy } \\
\hline Exudative & 27 & 2 & 5 \\
\hline CMO noted & 0 & 2 & 0 \\
\hline Ischaemic & 0 & 0 & 1 \\
\hline Vitreous haemorrhage & 2 & & \\
\hline
\end{tabular}

CWS, cotton wool spots; IRMA, intraretinal microvascular haemorrhage; NVD, neovascularisation of the optic disc; NVE, neovascularisation elsewhere: CMO. cystoid macular oedema.

"Sixty-five eyes had only background retinopathy.

${ }^{\text {t}}$ Three eyes had minimal changes only.

'This eye had a vitreous haemorrhage.

though these were all minimal with only a few scattered exudates in the macular area and none was sufficient to have been given treatment after clinical examination. It also missed 1 case of ischaemic maculopathy. Clinical examination missed 2 cases of cystoid macular oedema (CMO) which were detected by the SLO.

Several of the above features co-existed in a number of eyes; for example, eyes with new vessels also had preproliferative signs such as venous beading. Following SLO examination of each patient, a decision was made as to whether referral for clinical examination would have been necessary. Eighteen recommendations for referral were made, including the 3 patients ( 5 eyes) in whom the SLO view was too poor to use. Of these, 14 patients did need laser treatment, 5 for proliferative retinopathy and 8 for exudative maculopathy. Of those not requiring treatment, 1 had regressing peripheral new vessels, 1 had a small area of IRMA but no other clinical evidence of ischaemia, and the remaining 2 patients ( 4 eyes) were referred because of the poor SLO image. One of these patients was listed for cataract surgery. All patients and all eyes requiring treatment would have been referred.

\section{DISCUSSION}

In order to be a useful screening tool in diabetic retinopathy, the SLO should be simple enough to use, be cost-effective, must not miss retinopathy requiring treatment or closer monitoring (high sensitivity), and must not cause the referral of too many unnecessary cases for further examination (low specificity). ${ }^{2}$ Whilst it is not as mobile or as simple to use as the non-mydriatic fundus camera, an ophthalmic photographer can readily become competent in its use. It is expensive to purchase, but after the initial capital outlay the running costs are low: apart from the photographer's time and the reporting time, the only cost is the U-Matic videotape which can store results 
from 30 patients on one cassette and which can be re-used if a permanent record is not required after the images have been reported. If desired a print-out can be made from the frame-grabber which is an integral part of the equipment.

This study set out to find how reliable the SLO was at detecting retinopathy. There has been concern with nonmydriatic fundus cameras that they could miss new vessels, particularly those which were growing forwards into the vitreous as they would not be in focus. ${ }^{6}$ The SLO has a much greater depth of field and such vessels ought not to be missed. At first it seemed disappointing that any new vessels had been missed, but when the relevant cases were examined more closely it was apparent that this only applied to regressing or inactive vessels (i.e. the vessels had been previously noted, and following laser treatment were either static or regressing leaving a mainly fibrotic remnant), the only exception being a patient with a vitreous haemorrhage - a reason for clinical review anyway. The SLO missed 4 cases of new vessels at the optic disc, but on checking the clinical data 3 of these were inactive vessels following laser treatment, and the fourth was the patient with a vitreous haemorrhage. The SLO missed 1 area of new vessels elsewhere but these too were inactive. Therefore there were no eyes with active new vessels which would not have been referred. In 2 eyes the SLO detected new vessels which were missed by conventional examination but which were subsequently confirmed clinically. It may be that the SLO is more useful than clinical examination alone in assessing new vessels after laser treatment to see whether they are still active; that is, if they fail to show well on the SLO this may signify regression, saving unnecessarily frequent clinic visits to monitor vessels or to undergo fluorescein angiography. This is the subject of a separate study. It is important to note that in 2 cases the SLO detected active new vessels which were missed on clinical examination but which were confirmed subsequently.

Preproliferative changes were not seen so easily, and in particular cotton wool spots were missed in several patients. However, the authors have found that with more experience these are becoming easier to identify. The IRMA that were missed were both very subtle changes.

The study could be criticised for not utilising flourescein angiography to confirm some findings, in particular $\mathrm{CMO}$, but the research team have become used to the appearance of CMO with the SLO and now find that fluorescein angiography is largely unnecessary in its diagnosis. ${ }^{9}$

An important feature of any screening test is that most if not all patients can be examined. In some studies with the non-mydriatic fundus camera, $17-23 \%$ of photographs were unusable. ${ }^{3.6}$ In this study, the SLO views of $4.63 \%$ of patients ( 5 eyes) were unusable, but in 2 of these eyes there was a cataract sufficiently dense to require surgery, and in a third there was a vitreous haemorrhage, itself prompting referral. Therefore the SLO views of only 2 eyes (1.9\%) were unusable for technical reasons. Of those views which were poor but usable, 2 eyes with fairly advanced cataract (1 patient) were examined more easily with the SLO than by conventional examination and that patient underwent urgent cataract surgery. The use of a scanning spot system by the SLO means that, unlike conventional photography, it does not depend on the optics of the eye to produce a focused image, and so it can obtain a good image in cataractous eyes unless the cataract is completely opaque. ${ }^{10} \mathrm{Of}$ the other unusable images, 4 eyes (2 patients) had very small pupils and could probably have been examined easily with the SLO if they had been dilated with tropicamide.

From this study it is suggested that diabetic patients could safely be examined using the SLO as a screening tool to detect changes in retinopathy and not just for a baseline screen. Patient compliance with follow-up would be improved as, with few exceptions, patients would require no mydriasis, and they could attend whenever the photographer was available rather than having to attend a specific ophthalmologist's clinic. However, the videotape would still need to be viewed by an experienced clinician.

Key words: Diabetic retinopathy, Scanning laser ophthalmoscope, Screening.

\section{REFERENCES}

1. Ulbig MRW, Hamilton AMP. Factors influencing the natural history of diabetic retinopathy. Eye 1993;7:242-9.

2. MacCuish AC. Early detection and screening for diabetic retinopathy. Eye 1993;7:254-9.

3. Williams R, Nussey S, Humphrey R, Thompson G. Assessment of non-mydriatic fundus photography in detection of diabetic retinopathy. BMJ 1986;293:1140-2.

4. Burns-Cox CJ, Dean Hart JC. Screening of diabetics for diabetic retinopathy by ophthalmic opticians. BMJ 1985; 290:1052-4.

5. Bron AJ. Screening for treatable diabetic retinopathy. BMJ 1985;290:1025-6.

6. Jones D, Dolben J, Owens DR, Vora JP, Young S, Creagh FM. Non-mydriatic Polaroid photography in screening for diabetic retinopathy: evaluation in a clinical setting. BMJ 1988;296:1029-30.

7. Mohan R, Kohner EM, Aldington SJ, Nijar I, Mohan V, Mather HM. Evaluation of a non-mydriatic camera in Indian and European diabetic patients. Br J Ophthalmol 1988; $72: 841-5$.

8. Webb RH, Hughes GW, Delori FC. Confocal scanning laser ophthalmoscope. Appl Optics 1987;26:1492-9.

9. Wykes WN, McLaughlin E. Living with a scanning laser ophthalmoscope. Eye 1992;6:535-6.

10. Mainster MA, Timberlake GT, Webb RH, Hughes GW. Scanning laser ophthalmoscopy: clinical applications. Ophthalmology 1982;89:852-7. 